\title{
Abstract: Deep Transfer Learning for Aortic Root Dilation Identification in 3D Ultrasound Images
}

\author{
Jannis Hagenah ${ }^{1}$, Mattias Heinrich ${ }^{2}$, Floris Ernst ${ }^{1}$ \\ ${ }^{1}$ Institut für Robotik und Kognitive Systeme, Universität zu Lübeck \\ ${ }^{2}$ Institut für Medizinische Informatik, Universität zu Lübeck \\ hagenah@rob.uni-luebeck.de
}

Valve-sparing aortic root reconstruction presents an alternative to valve replacement. However, choosing the optimal prosthesis size for the individual patient is a critical task during surgery. To assist the surgeons in their decision making, a pre-operative surgery planning tool based on 3D ultrasound data has been proposed. One step in the workflow is the automatic discrimination of healthy and pathologically dilated aortic roots. Up to date, hand-crafted features were extracted from the images for this purpose of training a classifier. A study showed the limited classification accuracy of this method, indicating that feature learning would present a promising alternative. However, training deep neural networks requires large datasets.

In this work, we propose transfer learning to use image features derived by deep neural networks on the available very small data sets [1]. For this purpose, we used the pretrained deep neural network VGG16. We used the activation of the last convolutional layer as extracted features of the input image.

To simplify the problem, we manually identified two prominent horizontal slices through the ultrasound volume: The coaptation plane and the commissure plane. We propagated both images through the network and stitched the resulting features together to describe one aortic root sample. We did this for the whole data set of 48 images (24 healthy, 24 dilated). On the resulting dataset, we trained a Random Forest classifier (400 trees) and evaluated the classification accuracy using 10-fold cross validation.

Using the transferred deep features we could reach a classification accuracy of $84 \%$, which clearly outperformed the hand-crafted features (71\% accuracy). Adding the hand-crafted features to the transferred ones did not increase the accuracy $(83 \%)$ Hence, all of the information contained in the hand-crafted features can be provided using transfer learning. Even though the VGG16 network was trained on RGB photos and different classification tasks, the learned feaures are still relevant for ultrasound image analysis of aortic root pathology identification. Hence, transfer learning makes deep learning possible even on very small ultrasound data sets.

\section{References}

1. Hagenah J, Mattias H, Floris E. Deep transfer learning for aortic root dilation identification in 3D ultrasound images. Curr Dir Biomed Eng. 2018;4.1:71-74. 